hep-ph/0106356

SCIPP-01-26

\title{
Comments on Non-Commutative Phenomenology
}

\author{
Alexey Anisimov, Tom Banks, Michael Dine and Michael Graesser \\ Santa Cruz Institute for Particle Physics, Santa Cruz CA 95064
}

\begin{abstract}
It is natural to ask whether non-commutative geometry plays a role in four dimensional physics. By performing explicit computations in various toy models, we show that quantum effects lead to violations of Lorentz invariance at the level of operators of dimension three or four. The resulting constraints are very stringent.
\end{abstract}




\section{Introduction}

Mathematicians proposed non-commutative geometry and the corresponding non-commutative field theories as a possible alternative to conventional field theories [1]. Physicists, for some time, were skeptical about the possible role of such theories, given their non-local character. However, interest increased with the realization that such theories can appear as the low energy limit of string theory in certain regions of the moduli space[2]. This has lead a number of authors, to ask whether such non-commutativity might play a role in physics, and particularly whether non-commutative field theory might become manifest at accessible energy scales [3, 4, 5, 7, 8, 9]. Unlike the situation for supersymmetry or large dimensions, we do not currently possess a compelling argument that non-commutativity should be relevant, or that it should be relevant at some particular energy scale. Indeed, it is not clear that we can write down a non-commutative generalization of the standard model in a simple way. Still, one can ask whether we can constrain any possible non-commutativity in four dimensions from present experiments. The most striking feature of such non-commutativity is likely to be the violation of Lorentz invariance.

The authors of [8], for example, have investigated the violations of Lorentz invariance which would arise in a non-commutative version of QED. To proceed, these authors take the tree level lagrangian of the theory, and perform the Seiberg-Witten map to rewrite the theory in terms of conventional quantum fields. This generates a number of Lorentz-invariance violating operators, all with two derivatives relative to the renormalizable terms, i.e. dimension six. This is basically because the indices on $\theta_{\mu \nu}$ must be contracted with derivatives. They argue that these constrain the parameter $\theta$ to be of order $\left(1-10^{-2}\right) \mathrm{TeV}^{-2}$. Far stronger bounds arise if one considers potential non-commutative effects in strong interactions [4].

On the other hand, one can imagine that operators of lower dimension, such as

$$
\mathcal{O}_{1}=m_{e} \theta^{\mu \nu} \bar{\psi} \sigma_{\mu \nu} \psi \quad \mathcal{O}_{2}=\theta^{\mu \nu} \bar{\psi} D_{\mu} \gamma_{\nu} \psi \quad \mathcal{O}_{3}=\left(\theta^{2}\right)^{\mu \nu} F_{\mu \rho} F_{\nu}^{\sigma} \quad \mathcal{O}_{4}=\theta^{\mu \nu} \theta^{\rho \sigma} F_{\mu \nu} F_{\rho \sigma}
$$

might appear at some level. Indeed, we will demonstrate in this paper that these operators are generated by quantum effects, in a variety of non-commutative theories. Typically they are generated at two loop order. Because these operators are (effectively) dimension four, while $\theta$ has dimensions of inverse mass-squared, the coefficient must involve additional dimensionful factors. As we will see, if we suppose that the theory has some cutoff, $\Lambda$, then there are two interesting regimes. In the first, $\theta \Lambda^{2} \gg 1$, and the additional dimensions are just made up by factors of $\operatorname{Tr}\left(\theta^{2}\right)$. In other words, the coefficients are independent of the magnitude of $\theta$ ! In this case, such non-commutativity is ruled out, no matter what the scale at which it might be 
relevant. This is an example of the infrared-ultraviolet connection.

On the other hand, such a cutoff is probably not sensible for other reasons, connected with the infrared-ultraviolet connection. For example, scalar propagators have bizarre infrared singularities 10]. In the limit $\theta \Lambda^{2} \ll 1$, the coefficients of these operators are proportional to this dimensionless quantity. The limits on such operators are quite stringent. For example, $\mathcal{O}_{1}$ has the structure of a coupling of the electron magnetic moment to a background magnetic field (though there is no corresponding orbital coupling). Just given that one can detect the earth's magnetic field with ordinary magnets, one should be able to establish a limit of order $10^{-15}$ on its dimensionless coefficient. Indeed, the actual limits are orders of magnitude more stringent, particularly from clock comparison tests [14] and from spin-polarized solids [11]. $\mathcal{O}_{2}$ would lead to propagation of photons with different speeds depending on their polarizations. Again, stringent limits exist, here from cosmic birefringence [21]. However, because the coefficient is quadratic in $\theta$, the corresponding limits on $\theta \Lambda^{2}$ are significantly weaker.

In any case, even in this limit, if we combine the limit on $\theta \Lambda$ with plausible restrictions on $\Lambda$, we can set very strong limits on $\theta$. For example, if $\Lambda=1 \mathrm{TeV}$, than calling $\theta=1 / M^{2}$, we will see $M>10^{12-13} \mathrm{GeV}$, or possibly stronger. The limit scales linearly with $\theta$.

\section{Operators Involving Photons}

We consider first operators of the type $\mathcal{O}_{3}$. These are generated already at one loop, in a non-commutative version of QED. Related computations have been done in [6]. The required Feynman rules can be found there, and in [5]. A straightforward computation gives, for the vacuum polarization:

$$
i \Pi_{\mu \nu}(q)=-4 e^{2} \int \frac{d^{4} k}{(2 \pi)^{4}} \sin ^{2}(k \wedge q)\left[4 k_{\mu} k_{\nu}+\left(k^{2} g_{\mu \nu} \text { pieces }\right)\right] \frac{1}{k^{4}}
$$

where, noting that we will be interested in the leading terms at small momentum, we have suppressed the $q$ dependence except that from the Moyal factors. If we suppose that the cutoff in the theory is much larger than $1 / \theta$, then this expression, while ultraviolet finite, is singular at small momenta. Instead, we can consider the limit $\theta \Lambda^{2} \ll 1$. Now we have to ask precisely how we cut off the theory in a gauge-invariant fashion. We will not investigate this question carefully here円. Instead, we note that the operator $\mathcal{O}_{4}$ receives a contribution from the first term in the

\footnotetext{
${ }^{1}$ One approach to a cutoff starts with the observation of [6] that in a supersymmetric theory, this contribution is canceled by the contribution of gauginos. If we introduce a soft mass for the gauginos, then, this could act as a regulator.
} 
integrand, and this contains terms that are at least formally gauge invariant. Introducing a momentum-space cutoff yields:

$$
\mathcal{L}_{e f f} \approx-\frac{e^{2}}{16 \pi^{2}} \Lambda^{4} \mathcal{O}_{4}
$$

So already at one loop, Lorentz-invariant dimension four terms are present. As we will see, the experimental limits on such terms are impressive. But limits on the operator $\mathcal{O}_{1}$ are potentially much stronger, given that it depends linearly on $\theta$. In the next section, we study this operator.

\section{Two loop Contributions to the Fermion Lagrangian}

We consider a Yukawa theory, with lagrangian,

$$
\mathcal{L}=i \bar{\psi} \not \partial \psi+\frac{1}{2}(\partial \phi)^{2}+g \bar{\psi} \star \phi \star \psi-m \bar{\psi} \psi
$$

It is important here that $\psi$ is a Dirac fermion; otherwise the coefficient of the would-be operator, $\mathcal{O}_{1}$, vanishes [2. The Feynman rules for this theory are given, for example, in [10]. To study the operator $\mathcal{O}_{1}$, we study the fermion self energy evaluated on-shell. At one loop, the only diagram is planar, and there is no $\theta$-dependence. At two loops, however, there is a non-planar diagram. This diagram has non-trivial $\theta$-dependence. In the limit that $\theta \Lambda^{2} \ll 1$, we can expand the integrand in powers of $\theta$. There is a term proportional to $\sigma_{\mu \nu}$ which is quadratically divergent: ${ }^{3}$

$$
4 m \int \frac{d^{4} k}{(2 \pi)^{4}} \frac{d^{4} \ell}{(2 \pi)^{4}} \ell \wedge k \not k \downarrow \frac{1}{k^{4}(k+\ell)^{4}(\ell)^{2}},
$$

where $\ell \wedge k \equiv \ell_{\mu} \theta^{\mu \nu} k_{\nu}$. Simplifying, this yields:

$$
4 m i \int \frac{d^{4} k}{(2 \pi)^{4}} \frac{d^{4} \ell}{(2 \pi)^{4}} k^{\mu} k^{\nu} \ell^{\rho} \ell^{\sigma} \theta_{\mu \rho} \sigma_{\nu \sigma} \frac{1}{k^{4}(k+\ell)^{4}(\ell)^{2}}
$$

To perform the integral, it turns out to be simplest to first combine the first two terms using a Feynman parameter. This yields

$$
24 i m \int \frac{d^{4} k d^{4} \ell}{(2 \pi)^{8}} d x \frac{x(1-x) k \wedge \ell k_{\mu} \ell_{\nu} \sigma^{\mu \nu}}{\left[k^{2}+\ell^{2} x(1-x)\right]^{4} \ell^{2}}
$$

\footnotetext{
${ }^{2}$ This is because the phase factor appearing at the vertex is a cosine for a Majorana fermion, but an exponential for a Dirac fermion.

${ }^{3}$ Here and below, we do not carefully distinguish the operator $\mathcal{O}_{1}$ from operators such as $\theta^{\mu \nu} \bar{\psi} \sigma_{\mu \nu} \not D \psi$, which are equivalent if one uses the equations of motion. We will confine our attention here to on-shell computations.
} 
One can now do the integral over $k$, leaving

$$
\frac{m}{2 \pi^{2}} \frac{1}{\left(16 \pi^{2}\right)^{2}} \frac{\theta^{\mu \nu} \sigma_{\mu \nu}}{\ell^{2}}
$$

so, introducing a simple momentum space cutoff $\Lambda^{2}$ on $\ell^{2}$, we obtain

$$
\mathcal{L}_{e f f}=\frac{1}{2} m \Lambda^{2}\left(\frac{g^{2}}{16 \pi^{2}}\right)^{2} \theta^{\mu \nu} \bar{\psi} \sigma_{\mu \nu} \psi .
$$

The main point here is that the result is non-zero, and, up to factors of order one, is of the size one might naively guess.

In the limit $\theta \Lambda^{2} \gg 1$, one can also easily obtain the leading piece of the integral. One now must keep the full exponential factor, $e^{i k \wedge \ell}$. However, in some ways, this is simpler. We now have to study

$$
\int d^{4} k d^{4} \ell \frac{4 m k_{\mu} \ell_{\nu} \sigma^{\mu \nu} e^{i k \wedge \ell}}{k^{4}(k+\ell)^{4} \ell^{2}}
$$

Although by simple power counting this integral is both ultra-violet and infra-red divergent, the dependence on $\theta_{\mu \nu}$ is finite. To see this, first regulate the infra-red divergence by temporarily inserting an infra-red cutoff. For low momentum we may then expand the exponential to obtain a power series in $\theta$. The leading term is independent of $\theta$ and is divergent. But the integral for the subsequent terms depending on $\theta$ are convergent due to the additional powers of the momenta. For these terms we may remove the cutoff. In the ultra-violet the phase factor damps the logarithmic divergence, and again the $\theta$ dependence is finite.

This integral also has the interesting feature that it is independent of the overall scale of $\theta_{\mu \nu}$. To see this, write $\theta_{\mu \nu}=\theta b_{\mu \nu}$ where $b_{\mu \nu}$ is a matrix of numbers describing the orientation of $\theta_{\mu \nu}$ relative to a fixed coordinate system. By rescaling the momenta appearing in the integral the dependence on $\theta$ can be eliminated. Remarkably, our result in the limit of a large cutoff depends only on the direction of $\theta_{\mu \nu}$ and not its magnitude.

Now in the integral, we can proceed much as we did before, and obtain an analytic result in a few lines of algebra. First simplify the integral by setting the non-commutativity to lie, say, in the 1,2 direction. Then introduce a Feynman parameter to combine the first two factors in the denominator. One then shifts $k$ in the usual way, and obtains

$$
24 m \int \frac{d x d^{4} k d^{4} \ell x(1-x) \sigma_{\mu \nu} k^{\mu} l^{\nu}}{\left[k^{2}+\ell^{2} x(1-x)\right]^{4} \ell^{2}} e^{i \theta_{12}\left(k_{1} \ell_{2}-\ell_{1} k_{2}\right)} .
$$

Despite the shift the numerator retains the same form as before because $\sigma_{\mu \nu}$ and $\theta_{\mu \nu}$ are antisymmetric. The integral involving components of $\sigma_{\mu \nu}$ other than $\sigma_{12}$ now vanish. This is 
because only for the 1,2 components is the integrand not an odd function of the momenta. Then (11) simplifies to

$$
24 m \sigma_{12} \frac{1}{i} \frac{d}{d \theta} \int \frac{d x d^{4} k d^{4} \ell x(1-x)}{\left[k^{2}+\ell^{2} x(1-x)\right]^{4} \ell^{2}} e^{i \theta_{12}\left(k_{1} \ell_{2}-\ell_{1} k_{2}\right)} .
$$

Now one can rescale $\ell$ to eliminate the $x(x-1)$ in the denominator, and combine the remaining two factors with a new Feynman parameter. One can then integrate over the components of $k$ and $\ell$ in the 0 and 3 directions. Introducing a Schwinger parameter to exponentiate the remaining denominator, one can sequentially do the integrals over the 1 and 2 components of $k$ and $\ell$. All of the integrals involved are elementary, and one obtains, finally:

$$
\mathcal{L}_{\text {eff }}=\frac{4}{3} m\left(\frac{g^{2}}{16 \pi^{2}}\right)^{2} \frac{\pi \theta^{\mu \nu}}{\sqrt{\frac{1}{2} \operatorname{Tr} \theta^{2}}} \bar{\psi} \sigma_{\mu \nu} \psi
$$

These results are readily extended to $U(1)$ and $U(N)$ gauge theories containing fundamental matter. For the case of a $U(1)$ theory, there are two diagrams at two loop order 1 . The Feynman rules may be found in [5], after correcting a sign in the phase of the photon-electron-electron Feynman rule so that the vertex factor is proportional to $e^{i p_{I} \wedge p_{F}}$, for incoming (outgoing) electron momentum $p_{I}\left(p_{F}\right)$ and using the $\wedge$ notation defined below (5). One finds that these two diagrams contribute equally but add constructively, in both limits. In the case of a U(N) gauge theory, there are again two diagrams. The Feynman rules are given, for example, in [16] ๒. Here one also does not find a cancellation [. In fact the result is independent of $N$, so the $U(N)$ and $U(1)$ theories generate the operator with the same coefficient. One finds in the limit $\theta \Lambda^{2} \ll 1$ the effective Lagrangian for the on-shell amplitude is

$$
\mathcal{L}_{\text {eff }}=\frac{3}{4} m \Lambda^{2}\left(\frac{e^{2}}{16 \pi^{2}}\right)^{2} \theta^{\mu \nu} \bar{\psi} \sigma_{\mu \nu} \psi,
$$

where the $U(1)$ generator is normalized to $1 / 2$. In the limit $\theta \Lambda^{2} \gg 1$ it is given by

$$
\mathcal{L}_{e f f}=2 m\left(\frac{e^{2}}{16 \pi^{2}}\right)^{2} \frac{\pi \theta^{\mu \nu}}{\sqrt{\frac{1}{2} \operatorname{Tr} \theta^{2}}} \bar{\psi} \sigma_{\mu \nu} \psi \text {. }
$$

Finally, we can consider a theory with Yukawa interactions and a U(1) gauge interaction. In this case, there is again a non-zero contribution. Proceeding as above, we obtain:

$$
\mathcal{L}_{\text {eff }}=-m \Lambda^{2}\left(\frac{e g}{16 \pi^{2}}\right)^{2} \theta^{\mu \nu} \bar{\psi} \sigma_{\mu \nu} \psi
$$

\footnotetext{
${ }^{4}$ Two-loop diagrams with one-loop self-energy subgraphs do not contribute to $\mathcal{O}_{1}$.

${ }^{5}$ In this reference the momenta are pointing into the vertices. This is confirmed by taking the commutative limit to obtain the usual rule for non-abelian gauge theory.

${ }^{6} \mathrm{In}$ an earlier version a cancellation for both the $U(1)$ and $U(N)$ theories was obtained. This result was incorrect due to a sign error in deriving a Feynman rule.
} 
where $e$ and $g$ denote the gauge and Yukawa couplings, respectively. In a $\mathrm{U}(\mathrm{N})$ gauge theory this is generalized to

$$
\mathcal{L}_{e f f}=-\frac{N}{2} m \Lambda^{2}\left(\frac{e g}{16 \pi^{2}}\right)^{2} \theta^{\mu \nu} \bar{\psi} \sigma_{\mu \nu} \psi
$$

We will discuss the possible experimental implications of these results below.

These calculations have all been done in terms of the non-commutative variables. Because we have calculated operators which are bilinear in the fields, the non-commutativity is not relevant. We have considered how these computations might look if one first performs the Seiberg-Witten map. Indeed, the calculations are distinctly more complicated. At one loop, it is obvious that there is no $\theta$-dependence in the self energy, if one works with the noncommutative variables. If, in a $U(1)$ theory, say, one first performs the Seiberg-Witten map, there are many individual diagrams with non-trivial $\theta$ dependence. Off shell, working, say, at zero momentum, it is easy to see that there are terms in the effective action with non-trivial $\theta$-dependence. We have checked that the $\theta$-dependence vanishes on shell. This seems quite reasonable. Thinking of the Seiberg-Witten transformation as a field redefinition, we do not expect correlation functions of the new fields to be the same as those of the original ones, but we do expect on-shell quantities to be the same.

In general, then, we see that the operator $\mathcal{O}_{1}$ is generated. In the small $\Lambda \theta$ limit, we expect the experimental bounds to be very strong. We will discuss these bounds in the next section. One might object that the cutoff we have introduced is artificial. A natural cutoff for noncommutative field theory is to realize it as a large $N$ limit of lower dimensional field theories of $N \times N$ matrices (though the precise nature of the limit has not yet been understood). In this way one appears to guarantee that counterterms will all involve $*$-products. However, precisely in the two loop nonplanar graphs that we have studied, one encounters graphs that produce double trace operators. These are the diagrams that exhibit UV-IR mixing reminiscent of the exchange of closed string states in open string field theory. It is important then, that we have done the two loop calculation in the infinite cutoff limit as well, and found a relevant operator with a coefficient independent of the scale of $\theta$. One can easily imagine that UV-IR mixing will produce all sorts of bizarre effects that might lead to experimental signatures. This has not been studied. Our results show that in addition to these peculiar effects, the NC field theory produces a relevant Lorentz violating term with large coefficient. The term is precisely of the form we found with an ad-hoc cutoff much lower than the energy scale of noncommutivity. Thus, the constraints on a noncommutative field theory with no cutoff are much more stringent than those we will exhibit in the next section. 


\section{Experimental limits}

One expects that there are extraordinary limits on the coefficient of the operator $\mathcal{O}_{1}$. For example, from the fact that one can measure the earth's magnetic field with a compass, one can set a limit on the coefficient of $\mathcal{O}_{1}$ of order $10^{-15} \mathrm{MeV}$. From precision measurements in atomic systems, one expects to be able to set very strong limits. In fact, the best limit on this operator for the electron comes from magnetic systems [11], where a limit of approximately $10^{-25} \mathrm{MeV}$ is set [12]. This bound is obtained from studying the oscillation of a highly electron spin-polarized torsion pendulum, where the presence of the operator $\mathcal{O}_{1}$ and the rotation of the earth induces a time-dependent macroscopic torque on the pendulum [13, 12]. The limit from precision tests of hyperfine splitting is about one order of magnitude weaker [14].

A number of authors have studied limits on dimension six operators proportional to $\theta$. In non-commutative QED, one can set limits on $\sqrt{\theta}$ in the several TeV range[ [8]. The strongest limit is that discussed in [⿶]. These authors assume that $\theta$-dependent terms in a non-commutative version of QCD lead to a coupling $\theta^{\mu \nu} \bar{N} \sigma_{\mu \nu} N$, where $N$ is the nucleon wave function. This leads to a striking limit on $\theta, \sqrt{\theta}<\left(10^{15} \mathrm{GeV}\right)^{-1}$.

If we attempt to take the cutoff to infinity, it is clear that we can rule out non-commutativity at any scale. In this limit the coefficients of the Lorentz-violating operators are far too large to accommodate the experimental bounds, for they are suppressed only by loop and gauge or Yukawa factors and are independent of the size of the non-commutative scale. This is a reflection of the infrared-ultraviolet connection. On the other hand, as in [7], it is more reasonable to include an explicit cutoff. Otherwise, one will obtain, for example, unacceptable infrared behavior for gauge boson and scalar propagators.

Lacking a complete non-commutative generalization of the standard model, it is hard to set precise limits. Still, we have seen that in theories with Yukawa and gauge couplings, there are contributions to $\mathcal{O}_{1}$ which proportional to $\alpha y^{2}$, where $y$ is the Yukawa coupling, and contributions proportional to $\alpha^{2}$. The latter contributions are identical in both the pure gauge $U(N)$ and $U(1)$ theories. This suggests the possibility, although unlikely, that in a noncommutative formulation of $S U(N)$ the two-loop pure gauge contribution could vanish. To verify or disprove this speculation would require a computation in a satisfactory formulation of $S U(N)$ non-commutative theories. Given these remarks, we make the conservative choice of obtaining bounds from only the pure $U(1)_{e m}$ and mixed gauge-Yukawa contributions and ignore the much stronger bounds obtained from the pure $U(3)_{c}$ contribution. 
For the electron, in particular, the contributions proportional to $\alpha_{e m} \lambda^{2}$ translate to a limit on the dimensionless quantity $\theta \Lambda^{2}$, roughly:

$$
\theta \Lambda^{2}<10^{-8}
$$

There are also much larger contributions proportional to $\alpha_{e m}^{2}$. For the electron, these translate to a limit on the dimensionless quantity $\theta \Lambda^{2}$, roughly:

$$
\theta \Lambda^{2}<10^{-19}
$$

Given that the operator is suppressed by a power of the electron mass, it might be advantageous to study Lorentz violating constraints in muons. A reanalysis of Zeeman hyperfine splitting data in muonium, looking for sideral time variations, could constrain the coefficient of $\mathcal{O}_{1}$ to be less than $10^{-19} \mathrm{MeV}$ [17]. Future data from the BNL $g-2$ experiment could improve the latter limit by two to three orders of magnitude [17]. Putting in the numbers, the latter limit would only constrain $\theta \Lambda^{2}$ at the same level as the torsion pendulum experiment, whereas the hyperfine data provide a weaker bound.

From the neutron and proton, we obtain a stronger limit. The precise value requires translating the quark moments to nuclear moments, and we will content ourselves with very crude estimates. The strongest experimental limit comes from the neutron [14, where the limit is $10^{-27} \mathrm{MeV}$. First consider the contributions proportional to the Yukawa couplings and the strong coupling. For the up and down quarks, this translates, roughly, to a limit on the dimensionless combination

$$
\theta \Lambda^{2}<10^{-17}
$$

We can do better using the strange quark. Here however we need to know what fraction of the nucleon spin is due to the strange quark, for which there is both considerable theoretical and experimental uncertainty. The SAMPLE collaboration has recently measured the strange contribution to the nucleon magnetic moment, and find $\mu_{s}=0.01 \pm 0.29 \pm 0.31 \mu_{N}$ [18], where $\mu_{N}$ is the Bohr magneton. Using the central value a rough limit is

$$
\theta \Lambda^{2}<10^{-19}
$$

Let us now consider the pure electromagnetic contributions. It is reasonable to expect that the matrix element of this contribution does not vanish for either the proton or neutron. Here though the experimental limit is weaker for the proton, with a bound of roughly $10^{-24} \mathrm{MeV}$ 
[14]. This translates into a limit

$$
\theta \Lambda^{2}<10^{-19}
$$

which is comparable to the bound obtained from the electron. The limit from the neutron is stronger, roughly :

$$
\theta \Lambda^{2}<10^{-22}
$$

A three-loop pure $S U(3)_{c}$ contribution provides a stronger limit. For the neutron we would obtain $\theta \Lambda^{2}<10^{-23}$. Limits on $\mathcal{O}_{2}$ involving the nucleon also exist, but are typically three orders of magnitude weaker than $\mathcal{O}_{1}$ [14]. A non-vanishing pure gauge contribution at two-loops, could be competitive with the limits found here. Work is in progress in evaluating the contribution to this operator [19].

We can also consider Lorentz violating operators involving the photon. Starting at two loops, for example, the following dimension 4 operators may be generated,

$$
\mathcal{O}_{3}=\lambda_{3}\left(\theta^{2}\right)_{\mu \nu}\left(F^{2}\right)^{\mu \nu} \quad, \mathcal{O}_{4}=\frac{\lambda_{4}}{8}\left(\theta_{\mu \nu} F^{\mu \nu}\right)^{2} \quad, \quad \mathcal{O}_{5}=\lambda_{5} \theta_{\mu \rho} F^{\rho \sigma} \theta_{\sigma \tau} F^{\tau \mu}
$$

The $\lambda_{i} \sim(\alpha / 4 \pi)^{2} \Lambda^{4}$ and their detailed values are unimportant for this discussion. These Lorentz-violating operators introduce $B_{i} B_{j}$ and $E_{i} E_{j}$ terms into the action, and they affect the propagation of light in vacuum, causing the vacuum to behave much like an anisotropic dielectric medium [20]. In particular, light travels at different speeds depending on the directions of polarization and propagation. This leads to cosmic birefringence, for which stringent limits already exist 21, 20].

To understand this, for simplicity consider $\mathcal{O}_{4}$ only. The behavior discussed here will also apply to the other operators. Assume that $\theta$ is non-zero in the 1-2 direction. Solving the field equations one finds that for light moving only in the 3-direction $p^{0}=\left|p_{3}\right|$. Similarly, light moving in either the 1 or 2 directions but polarized along the 3 direction is unaffected. But light moving along the 1-direction say, and polarized in the 2-direction has a modified dispersion relation, $p^{0}=\left(1+\lambda_{2} \theta^{2} / 2+\cdots\right)\left|p_{1}\right|$. The modification for more general polarizations and directions of propagation is easily worked out.

The experimental limit on this effect is obtained from studying polarized light from distant radio sources, and looking for a dependence of the angle of polarization on the distance to the source [21]. More specifically, 22] studied a large number of radio sources, and found a strong correlation between the angle of polarized light and the major axis of the source (after correcting 
for Faraday rotation). For the active radio sources this angle is roughly $90^{\circ}$, indicating that the magnetic fields of these sources runs parallel to the major axis, and that the light is produced by synchrotron radiation. If Lorentz violation is present, then the angle of the polarized light relative to the major axis will be rotated away from $90^{\circ}$ by an amount that grows with the distance to the source. Examining this data, Carroll, Field and Jackiw do not find any redshift dependence [21]. This constrains the relative phase given above to be $\phi=\delta \vec{p} \cdot \vec{x} \lesssim \mathcal{O}(1)$. Since the galaxies observed in [22] span a sizable fraction of the observable Universe, the constraint is

$$
\frac{|\delta \vec{p}|}{H} \lesssim \mathcal{O}(1) \Rightarrow \delta p \lesssim 10^{-42} \mathrm{GeV},
$$

with the limit quoted in [21] smaller by $h_{0} / 4$. Strictly speaking, 21] studied the effect of a different set of operators, namely those of the type $E_{i} B_{j}$. These lead to a dispersion relation that is independent of wavelength [21, 20], for which they obtain the bound quoted above. As [20] argue, we cannot directly translate this limit to a bound on the operators in (24) since the dispersion relation is no longer independent of wavelength, making it is more difficult to disentangle the Lorentz violating effect from Faraday rotation. In principle, however, these effects could be distinguished since the Faraday rotation depends on the square of the wavelength, whereas for the Lorentz violating effect the phase scales inversely with wavelength. Following [20], it is then reasonable that the data should still imply a bound that is roughly given by (25). As the wavelength of the radio sources analyzed in [22] are typically $10 \mathrm{~cm}$, inserting this value in the previous dispersion relation leads to the limit of

$$
\lambda_{i} \theta^{2}<10^{-28}
$$

This is an impressive limit, but because $\theta$ appears quadratically, it is not competitive with the terrestrial constraints. For inserting the loop factor suppression in $\lambda_{i} \sim(\alpha / 4 \pi)^{2} \Lambda^{4}$ leads to the bound

$$
\theta \Lambda^{2}<10^{-12}
$$

Of course, without a detailed underlying theory, these limits cannot be interpreted unambiguously. With plausible assumptions about the cutoff, we can bound $\theta$ at an extraordinary level. Even if the cutoff is $1 \mathrm{TeV}$, we can set a limit on $\theta, \theta<\left(10^{12-13} \mathrm{GeV}\right)^{-2}$.

\section{Acknowledgements:}


We thank A. Armoni, Jeff Harvey, Nemanja Kaloper and Scott Thomas, for conversations and comments. We also thank Mahiko Suzuki, who has similar observations, for stimulating discussions. This work supported in part by the U.S. Department of Energy.

\section{References}

[1] For a review with extensive references, see M.R. Douglas and N.A. Nekrasov, "NonCommutative Field Theory," hep-th/0106048, to appear in Reviews of Modern Physics.

[2] N. Seiberg and E. Witten, "String Theory and Non-Commutative Geometry," hepth/9908142, JHEP 09 (1999) 032.

[3] "Signals for Noncommutative Interactions at Linear Colliders", J. Hewett, F. Petriello, and T. Rizzo, hep-ph/001035 Phys.Rev.D64 :075012,2001.

[4] I. Mocioiu, M. Pospelov and R. Roiban, "Low Energy Limits on the Antisymmetric Tensor Field Background on the Brane and the Non-Commutative Scales," Phys. Lett. B489 (2000) 390, hep-ph/0005191.

[5] I. Riad and M.M. Sheikh-Jabbari, "Noncommutative QED and Anomalous Dipole Moments," hep-th/0008132.

[6] A. Matusis, L. Sussking, and N. Toumbas, "The IR/UV Connection in Noncommutative Gauge Theories", JHEP 0012 (2000) 002, hep-th/0002075.

[7] I. Hinchliffe and N. Kersting, "CP-violation from Noncommutative Geometry," hep$\mathrm{ph} / 0104137$.

[8] S. Carroll, J.A. Harvey, V. Alan Kostelecky, C.D. Lane and T. Okamoto, "Non Commutative Field Theory and Lorentz Violation," hep-th/0105082.

[9] Z. Guralnik, R. Jackiw, S.Y. Pi and A.P. Polychronakos, "Testing Non-Commutative QED, Constructing Non-commutative MHD", hep-th/0106044.

[10] S. Minwalla, M. Van Raamsdonk and N. Seiberg, "Non-Commutative Perturbative Dynamics," JHEP 0002 (2000) 20, hep-th/9912072.

[11] R. Bluhm and V. Alan Kostelecky, "Lorentz and CPT tests with Spin-Polarized Solids," Phys.Rev.Lett.84 (2000) 1381, hep-ph/9912542.

[12] M.G. Harris, Ph.D thesis, University of Washington, 1998.

[13] E.G. Adelberger et al., in P. Herczeg et al., eds., Physics Beyond the Standard Model, p. 717, World Scientific, Singapore, 1999.

[14] V.A. Kostelecky and C.D. Lane, "Constraints on Lorentz Violation from Clock-Comparison Experiments," Phys.Rev. D60 (1999) 116010, hep-ph/9908504. 
[15] M. Hayakawa, "Perturbative analysis on infrared and ultraviolet aspects of noncommutative QED on $R^{4 ", ~ h e p-t h / 9912167 . ~}$

[16] A. Armoni, "Comments on Perturbative Dynamics of Noncommutative Yang-Mills Theory", Nucl.Phys. B593 (2001) 229, hep-th/0005208.

[17] R. Bluhm, V.A. Kostelecky, and C. Lane, "CPT and Lorentz Tests with Muons", Phys. Rev. Lett. 84 (2000) 1098, hep-ph/9912451

[18] SAMPLE Collaboration (R. Hasty et. al., "Strange Magnetism and the Anapole Structure of the Proton", Science 290 (2000) 2117, nucl-ex/0102001.

[19] A. Anisimov, in progress.

[20] D. Colladay and V.A. Kostelecky, "Lorentz-Violating Extension of the Standard Model", Phys. Rev. D58 (1998) 116002, hep-ph/9809521.

[21] S. Carroll, G. Field and R. Jackiw, "Limits on a Lorentz and Parity Violation Modification of Electrodynamics", Phys. Rev. D41 (1990) 1231.

[22] P. Haves and R. G. Conway, Mon. Not. R. Astr. Soc. 173 (1975) 53; J. N. Clarke, P.P. Kronberg and M. Simard-Normandin, ibid., 190 (1980) 205. 